\title{
Approximation in law to the $d$-parameter fractional Brownian sheet based on the functional invariance principle
}

Xavier Bardina and Carme Florit

\begin{abstract}
We show a result of approximation in law of the $d$-parameter fractional Brownian sheet in the space of the continuous functions on $[0, T]^{d}$. The construction of these approximations is based on the functional invariance principle.
\end{abstract}

\section{Introduction}

The aim of this work is to give a result of convergence in law to the $d$-parameter fractional Brownian sheet. The approximations are a family of continuous processes constructed using the functional invariance principle.

The fractional Brownian motion of Hurst parameter $\alpha_{i} \in(0,1)$ is a centered Gaussian process $B^{\alpha_{i}}=\left\{B_{t}^{\alpha_{i}}, t \geq 0\right\}$ with the covariance function given by

$$
E\left(B_{t}^{\alpha_{i}} B_{s}^{\alpha_{i}}\right)=\frac{1}{2}\left(s^{2 \alpha_{i}}+t^{2 \alpha_{i}}-|t-s|^{2 \alpha_{i}}\right),
$$

(see, for instance, Mandelbrot and Van Ness (1968)).

The fractional Brownian motion can be extended to the multidimensional parameter space in two ways. One is the Lévy's fractional Brownian random field with parameter $\beta \in(0,1)$ (see Ciesielski and Kamont, 1995): a centered Gaussian process $Y$ with covariance function given by

$$
E\left(Y_{t} Y_{s}\right)=\frac{1}{2}\left(\|t\|^{2 \beta}+\|s\|^{2 \beta}-\|t-s\|^{2 \beta}\right),
$$

for $s, t \in \mathbb{R}^{d}$ and where $\|\cdot\|$ denotes the Euclidean norm.

2000 Mathematics Subject Classification: 60F17, 60G15, 60G60.

Keywords: $d$-parameter fractional Brownian sheet, weak convergence, functional invariance principle. 
The other extension of the fractional Brownian motion is the anisotropic fractional Wiener random field, introduced by Kamont (1996). This is a centered Gaussian process, defined on some probability space $(\bar{\Omega}, \overline{\mathcal{F}}, \bar{P})$, denoted by $B^{\alpha}=\left\{B_{s}^{\alpha}, s \in \mathbb{R}_{+}^{d}\right\}$, with covariance function given by

$$
\bar{E}\left(B_{s}^{\alpha} B_{t}^{\alpha}\right)=\prod_{i=1}^{d} \frac{1}{2}\left(s_{i}{ }^{2 \alpha_{i}}+t_{i}^{2 \alpha_{i}}-\left|t_{i}-s_{i}\right|^{2 \alpha_{i}}\right),
$$

where $\alpha=\left(\alpha_{1}, \alpha_{2}, \ldots, \alpha_{d}\right) \in(0,1)^{d}$. Observe that if $\alpha_{1}=\alpha_{2}=\cdots=\alpha_{d}=\frac{1}{2}$ we obtain the $d$-parameter Wiener process.

In this paper we will work with the second extension that we will call a $d$-parameter fractional Brownian sheet. In the section of preliminaries we will see that the $d$-parameter fractional Brownian sheet possesses a continuous version. It is possible to give a representation in law of $B^{\alpha}$ as an integral of a deterministic kernel with respect to a $d$-parameter Wiener process $W=\left\{W_{u} ; u \in[0, T]^{d}\right\}$ :

$$
\int_{0}^{t_{d}} \cdots \int_{0}^{t_{1}} K^{\alpha}(t, u) d W_{u}
$$

(see the next section for the definition of the kernel $K^{\alpha}$ ).

On the other hand, Donsker's theorem, known also as the functional invariance principle or the functional central limit theorem, states that the Wiener process can be approximated by a random walk constructed using a family of independent identically distributed random variables. More precisely, consider $\left\{Z_{k} ; k=\left(k_{1}, \ldots, k_{d}\right) \in \mathbb{N}^{d}\right\}$ an independent family of centered identically distributed random variables with variance equals to 1 . Then, the process

$$
n^{\frac{d}{2}} \int_{0}^{t_{d}} \cdots \int_{0}^{t_{1}} \sum_{k \in \mathbb{N}^{d}} Z_{k} I_{[k-1, k)}(u \cdot n) d u
$$

where $I_{[k-1, k)}(u \cdot n)=I_{\left[k_{1}-1, k_{1}\right) \times \cdots \times\left[k_{d}-1, k_{d}\right)}\left(u_{1} n, \cdots, u_{d} n\right)$, converges in law toward a $d$-parameter Wiener process (see, for instance, Wichura, 1969).

Using this result and representation (1.1) it is natural to try to approximate in law the process $B^{\alpha}$ by

$$
X_{n}(t)=n^{\frac{d}{2}} \int_{0}^{t_{d}} \cdots \int_{0}^{t_{1}} K^{\alpha}(t, u) \sum_{k \in \mathbb{N}^{d}} Z_{k} I_{[k-1, k)}(u \cdot n) d u .
$$

The main result of this work is that this family of processes converges in law, in the space of the continuous functions $\mathcal{C}\left([0, T]^{d}\right)$, to the $d$-parameter fractional Brownian sheet of parameters $\alpha=\left(\alpha_{1}, \ldots, \alpha_{d}\right)$. 
For the fractional Brownian motion, that is when $d=1$, the result is proved in Proposition 2.1 of Bardina et al. (2003) and also (for the Hurst parameter bigger than $\frac{1}{2}$ ) in a paper of Sottinen (2001). In this last paper the author construct, with this type of approximations, an elementary market model that converges weakly to the fractional analogue of the Black-Scholes model. There exist also other approximations for the fractional Brownian motion (see, for instance, Delgado and Jolis, 2000, Davydov, 1970, Pipiras and Taqqu, 2000 and Hult, 2003). Finally, for the 2-parameter fractional Brownian sheet, Bardina et al. (2003) prove a result of approximation in law for processes constructed from a Poisson process in the plane.

We have organized the paper as follows: the next section is devoted to some preliminaries and in Section 3 we prove our results.

As general rule the superscripts denote vectors of $\mathbb{N}^{d}$ or $\mathbb{R}^{d}$ and the subscripts the components of the vectors, e.g. $u^{j}=\left(u_{1}^{j}, \ldots, u_{d}^{j}\right)$. Positive constants, denoted by $C$, with possible subscripts indicating appropriate parameters, may vary from line to line.

\section{Preliminaries and notations}

Consider $[0, T]^{d} \subset \mathbb{R}^{d}$ with the usual partial order. Given $s, t \in[0, T]^{d}$ such that $s<t$ we denote by $\Delta_{s} X(t)$ the increment of the process $X$ over the rectangle $(s, t]=\prod_{i=1}^{d}\left(s_{i}, t_{i}\right) \subset \mathbb{R}^{d}$.

The fractional Brownian motion of Hurst parameter $\alpha_{i} \in(0,1), B^{\alpha_{i}}=$ $\left\{B_{t}^{\alpha_{i}}, t \in \mathbb{R}_{+}\right\}$admits an integral representation of the form (see for instance Alòs et al., 2001)

$$
B_{t}^{\alpha_{i}}=\int_{0}^{t} K_{\alpha_{i}}(t, s) d W_{s},
$$

where $W$ is a standard Brownian motion and the kernel $K_{\alpha_{i}}$ is defined on the set $\{0<s<t\}$ and given by

$$
\begin{aligned}
& K_{\alpha_{i}}(t, s)=d_{\alpha_{i}}(t-s)^{\alpha_{i}-\frac{1}{2}} \\
& \quad+d_{\alpha_{i}}\left(\frac{1}{2}-\alpha_{i}\right) \int_{s}^{t}(u-s)^{\alpha_{i}-\frac{3}{2}}\left(1-\left(\frac{s}{u}\right)^{\frac{1}{2}-\alpha_{i}}\right) d u,
\end{aligned}
$$

with $d_{\alpha_{i}}$ the following normalizing constant

$$
d_{\alpha_{i}}=\left(\frac{2 \alpha_{i} \Gamma\left(\frac{3}{2}-\alpha_{i}\right)}{\Gamma\left(\alpha_{i}+\frac{1}{2}\right) \Gamma\left(2-2 \alpha_{i}\right)}\right)^{\frac{1}{2}} .
$$


We can extend the kernels $K_{\alpha_{i}}$ over all $(0, T]^{2}$ by putting

$$
\tilde{K}_{\alpha_{i}}(s, x)= \begin{cases}K_{\alpha_{i}}(s, x) & \text { if } s>x \\ 0 & \text { if } s \leq x\end{cases}
$$

and for the sake of simplicity we will denote also by $K_{\alpha_{i}}$ these extensions.

Taking into account the expression (2.1) for the fractional Brownian motion we can consider the following integral representation for the $d$-parameter fractional Brownian sheet $B^{\alpha}$ :

$$
\int_{0}^{s_{d}} \cdots \int_{0}^{s_{1}} K_{\alpha_{1}}\left(s_{1}, u_{1}\right) K_{\alpha_{2}}\left(s_{2}, u_{2}\right) \cdots K_{\alpha_{d}}\left(s_{d}, u_{d}\right) d W_{u}
$$

where $s \in[0, T]^{d}, \alpha \in(0,1)^{d}$ and where $\left(W_{u}\right)_{u \in[0, T]^{d}}$ is a $d$-parameter Wiener process. Indeed, observe that this process is a centered Gaussian process with the same covariance function as $B^{\alpha}$. Moreover, the $d$-parameter fractional Brownian sheet possesses a continuous version. Using the Cencov's criteria (see Čencov, 1956) and that the process $B_{t}^{\alpha}$ is almost sure equal to zero when $t_{i}=0$ for some $i \in\{1, \ldots, d\}$ it is enough to prove that

$$
E\left(\left(\Delta_{s} B_{t}^{\alpha}\right)^{p}\right) \leq C\left[\left(t_{1}-s_{1}\right) \cdots\left(t_{d}-s_{d}\right)\right]^{1+\gamma},
$$

for some $\gamma>0$ and $p \geq 2$.

The increment $\Delta_{s} B_{t}^{\alpha}$ has a gaussian law with $E\left(\Delta_{s} B_{t}^{\alpha}\right)=0$ and, using the symmetry of the stochastic integral,

$$
\begin{aligned}
E\left(\left(\Delta_{s} B_{t}^{\alpha}\right)^{2}\right) & =E\left(\left(\int_{[0, T]^{d}} \prod_{i=1}^{d}\left(K_{\alpha_{i}}\left(t_{i}, u_{i}\right)-K_{\alpha_{i}}\left(s_{i}, u_{i}\right)\right) d W_{u}\right)^{2}\right) \\
& =\int_{[0, T]^{d}} \prod_{i=1}^{d}\left(K_{\alpha_{i}}\left(t_{i}, u_{i}\right)-K_{\alpha_{i}}\left(s_{i}, u_{i}\right)\right)^{2} d u \\
& =\prod_{i=1}^{d}\left(\int_{[0, T]}\left(K_{\alpha_{i}}\left(t_{i}, u_{i}\right)-K_{\alpha_{i}}\left(s_{i}, u_{i}\right)\right)^{2} d u_{i}\right) \\
& =\prod_{i=1}^{d} E\left(B_{t_{i}}^{\alpha_{i}}-B_{s_{i}}^{\alpha_{i}}\right)^{2}=\prod_{i=1}^{d}\left(t_{i}-s_{i}\right)^{2 \alpha_{i}},
\end{aligned}
$$

where $B^{\alpha_{i}}$ is a fractional Brownian motion of Hurst parameter $\alpha_{i}$.

Using the relation between the moments of a centered gaussian law we have that

$$
E\left(\left(\Delta_{s} B_{t}^{\alpha}\right)^{p}\right)=C_{p}\left(E\left(\left(\Delta_{s} B_{t}^{\alpha}\right)^{2}\right)\right)^{\frac{p}{2}},
$$

for all $p \geq 2$ even and this implies the inequality (2.4). 
Let $(\Omega, \mathcal{F}, P)$ be a probability space where we have defined $\left\{Z_{k} ; k \in\right.$ $\left.\mathbb{N}^{d}\right\}$, an independent family of identically distributed and centered random variables, with $E\left(Z_{k}^{2}\right)=1$ for all $k \in \mathbb{N}^{d}$, and such that $E\left(\left|Z_{k}\right|^{m}\right)<+\infty$ for all $k \in \mathbb{N}^{d}, m \in \mathbb{N}$.

Define for all $n \in \mathbb{N}$ and $t \in[0, T]^{d}$

$$
X_{n}(t)=n^{\frac{d}{2}} \int_{0}^{t_{d}} \cdots \int_{0}^{t_{1}} K^{\alpha}(t, u) \sum_{k \in \mathbb{N}^{d}} Z_{k} I_{[k-1, k)}(u \cdot n) d u
$$

where

$$
K^{\alpha}(s, u):=K_{\alpha_{1}}\left(s_{1}, u_{1}\right) K_{\alpha_{2}}\left(s_{2}, u_{2}\right) \cdots K_{\alpha_{d}}\left(s_{d}, u_{d}\right)
$$

and

$$
I_{[k-1, k)}(u \cdot n):=I_{\left[k_{1}-1, k_{1}\right) \times \cdots \times\left[k_{d}-1, k_{d}\right)}\left(u_{1} n, \cdots, u_{d} n\right) .
$$

We will also denote by $\theta_{n}$ the kernels

$$
\theta_{n}(u):=n^{\frac{d}{2}} \sum_{k \in \mathbb{N}^{d}} Z_{k} I_{[k-1, k)}(u \cdot n)
$$

So, we can write now that

$$
X_{n}(t)=\int_{[0, T]^{d}} K^{\alpha}(t, u) \theta_{n}(u) d u
$$

We will prove the convergence as $n$ tends to infinity, in the space of the continuous functions $\mathcal{C}\left([0, T]^{d}\right)$, of the laws of the family $X_{n}(t)$ to the law of the $d$-parameter fractional Brownian sheet.

The processes $X_{n}$ are continuous for every $\alpha \in(0,1)^{d}$ and absolutely continuous if $\alpha \in\left[\frac{1}{2}, 1\right)^{d}$. This is a consequence of the following lemma and the fact that $X_{n}(t)$ is equal to zero if $t_{i}=0$ for some $i \in\{1, \ldots, d\}$.

Lemma 2.1 Let $\theta \in L^{\infty}\left([0, T]^{d}\right)$ and define

$$
X(t)=\int_{[0, T]^{d}} K^{\alpha}(t, u) \theta(u) d u .
$$

Then,

$$
\left|\Delta_{s} X(t)\right| \leq C_{\alpha}\left(t_{d}-s_{d}\right)^{\left(\alpha_{d}+\frac{1}{2}\right) \wedge 1} \cdots\left(t_{1}-s_{1}\right)^{\left(\alpha_{1}+\frac{1}{2}\right) \wedge 1},
$$

for all $s, t \in[0, T]^{d}$ such that $s<t$. 
Proof: We have that,

$$
\begin{aligned}
\Delta_{s} X(t) & =\int_{[0, T]^{d}} \Delta_{s} K^{\alpha}(t, u) \theta(u) d u \\
& =\int_{[0, T]^{d}} \prod_{i=1}^{d}\left(K_{\alpha_{i}}\left(t_{i}, u_{i}\right)-K_{\alpha_{i}}\left(s_{i}, u_{i}\right)\right) \theta(u) d u \\
& \leq\|\theta\|_{\infty} \prod_{i=1}^{d} \int_{[0, T]}\left|K_{\alpha_{i}}\left(t_{i}, u_{i}\right)-K_{\alpha_{i}}\left(s_{i}, u_{i}\right)\right| d u_{i} .
\end{aligned}
$$

But,

$$
\begin{aligned}
\int_{[0, T]} \mid K_{\alpha_{i}} & \left(t_{i}, u_{i}\right)-K_{\alpha_{i}}\left(s_{i}, u_{i}\right) \mid d u_{i}= \\
& =\int_{[0, T]}\left|K_{\alpha_{i}}\left(t_{i}, u_{i}\right) I_{\left[0, t_{i}\right)}\left(u_{i}\right)-K_{\alpha_{i}}\left(s_{i}, u_{i}\right) I_{\left[0, s_{i}\right)}\left(u_{i}\right)\right| d u_{i} \\
& =\int_{s_{i}}^{t_{i}}\left|K_{\alpha_{i}}\left(t_{i}, u_{i}\right)\right| d u_{i}+\int_{0}^{s_{i}}\left|K_{\alpha_{i}}\left(t_{i}, u_{i}\right)-K_{\alpha_{i}}\left(s_{i}, u_{i}\right)\right| d u_{i} .
\end{aligned}
$$

Let us begin with the first summand of the last expression. If $\alpha_{i}<\frac{1}{2}$ then using (2.2) we have that

$$
K_{\alpha_{i}}\left(t_{i}, u_{i}\right) \leq C_{\alpha_{i}}\left(\left(t_{i}-u_{i}\right)^{\alpha_{i}-\frac{1}{2}}+\int_{u_{i}}^{t_{i}}\left(x-u_{i}\right)^{\alpha_{i}-\frac{3}{2}} d x\right),
$$

and

$$
\int_{s_{i}}^{t_{i}}\left|K_{\alpha_{i}}\left(t_{i}, u_{i}\right)\right| d u_{i} \leq C_{\alpha_{i}}\left(t_{i}-s_{i}\right)^{\alpha_{i}+\frac{1}{2}}
$$

On the other hand, when $\alpha_{i} \geq \frac{1}{2}$,

$$
\begin{aligned}
K_{\alpha_{i}}\left(t_{i}, u_{i}\right) & =C_{\alpha_{i}} u_{i}^{\frac{1}{2}-\alpha_{i}} \int_{u_{i}}^{t_{i}}\left(x-u_{i}\right)^{\alpha_{i}-\frac{3}{2}} x^{\alpha_{i}-\frac{1}{2}} d x \\
& \leq C_{\alpha_{i}} u_{i}^{\frac{1}{2}-\alpha_{i}} \int_{u_{i}}^{t_{i}}\left(x-u_{i}\right)^{\alpha_{i}-\frac{3}{2}} d x \\
& =C_{\alpha_{i}}\left(t_{i}-u_{i}\right)^{\alpha_{i}-\frac{1}{2}} u_{i}^{\frac{1}{2}-\alpha_{i}}
\end{aligned}
$$

and

$$
\int_{s_{i}}^{t_{i}}\left|K_{\alpha_{i}}\left(t_{i}, u_{i}\right)\right| d u_{i} \leq C_{\alpha_{i}}\left(t_{i}-s_{i}\right)^{\alpha_{i}-\frac{1}{2}} \int_{s_{i}}^{t_{i}} u_{i}^{\frac{1}{2}-\alpha_{i}} d u_{i} \leq\left(t_{i}-s_{i}\right) .
$$


For the second summand of (2.6), notice that if $\alpha_{i}=\frac{1}{2}$, then

$$
\int_{0}^{s_{i}}\left|K_{\alpha_{i}}\left(t_{i}, u_{i}\right)-K_{\alpha_{i}}\left(s_{i}, u_{i}\right)\right| d u_{i}=0 .
$$

When $\alpha_{i} \neq \frac{1}{2}$, we will use some bounds for the partial derivative of the kernel $K$. From (2.2) it is easy to check that the kernel $K_{\alpha_{i}}(t, s)$ is differentiable with respect to the first variable in the set $\{0<s<t\}$ and that

$$
\frac{\partial}{\partial t} K_{\alpha_{i}}(t, s)=d_{\alpha_{i}}\left(\alpha_{i}-\frac{1}{2}\right)\left(\frac{s}{t}\right)^{\frac{1}{2}-\alpha_{i}}(t-s)^{\alpha_{i}-\frac{3}{2}} .
$$

Then, for $\alpha_{i}>\frac{1}{2}$ we have that

$$
\left|\frac{\partial K_{\alpha_{i}}}{\partial t}(t, s)\right| \leq C_{\alpha_{i}} s^{\frac{1}{2}-\alpha_{i}}(t-s)^{\alpha_{i}-\frac{3}{2}}
$$

And so,

$$
\begin{aligned}
\int_{0}^{s_{i}}\left|K_{\alpha_{i}}\left(t_{i}, u_{i}\right)-K_{\alpha_{i}}\left(s_{i}, u_{i}\right)\right| d u_{i} & \leq \int_{0}^{s_{i}}\left(\int_{s_{i}}^{t_{i}}\left|\frac{\partial K_{\alpha_{i}}}{\partial r}\left(r, u_{i}\right)\right| d r\right) d u_{i} \\
& \leq C_{\alpha_{i}} \int_{0}^{s_{i}} u_{i}^{\frac{1}{2}-\alpha_{i}}\left(\int_{s_{i}}^{t_{i}}\left(r-u_{i}\right)^{\alpha_{i}-\frac{3}{2}} d r\right) d u_{i} \\
& \leq C_{\alpha_{i}}\left(t_{i}-s_{i}\right) \int_{0}^{s_{i}} u_{i}^{\frac{1}{2}-\alpha_{i}}\left(s_{i}-u_{i}\right)^{\alpha_{i}-\frac{3}{2}} d u_{i} \\
& =C_{\alpha_{i}} B\left(\frac{3}{2}-\alpha_{i}, \alpha_{i}-\frac{1}{2}\right)\left(t_{i}-s_{i}\right) .
\end{aligned}
$$

When $\alpha_{i}<\frac{1}{2}$ we have that

$$
\left|\frac{\partial K_{\alpha_{i}}}{\partial t}(t, s)\right| \leq C_{\alpha_{i}}(t-s)^{\alpha_{i}-\frac{3}{2}} .
$$

In this situation, we obtain the following majorization

$$
\begin{aligned}
\int_{0}^{s_{i}}\left|K_{\alpha_{i}}\left(t_{i}, u_{i}\right)-K_{\alpha_{i}}\left(s_{i}, u_{i}\right)\right| d u_{i} & \leq \int_{0}^{s_{i}}\left(\int_{s_{i}}^{t_{i}}\left|\frac{\partial K_{\alpha_{i}}}{\partial r}\left(r, u_{i}\right)\right| d r\right) d u_{i} \\
& \leq C_{\alpha_{i}} \int_{0}^{s_{i}}\left(\int_{s_{i}}^{t_{i}}\left(r-u_{i}\right)^{\alpha_{i}-\frac{3}{2}} d r\right) d u_{i} \\
& =C_{\alpha_{i}} \int_{s_{i}}^{t_{i}}\left(\left(r-s_{i}\right)^{\alpha_{i}-\frac{1}{2}}-r^{\alpha_{i}-\frac{1}{2}}\right) d r \\
& =C_{\alpha_{i}}\left(\left(t_{i}-s_{i}\right)^{\alpha_{i}+\frac{1}{2}}-t_{i}^{\alpha_{i}+\frac{1}{2}}+s_{i}^{\alpha_{i}+\frac{1}{2}}\right) \\
& \leq C_{\alpha_{i}}\left(t_{i}-s_{i}\right)^{\alpha_{i}+\frac{1}{2}} .
\end{aligned}
$$

The proof of the lemma is now complete. 


\section{Approximations in law}

The main result of this paper is the following theorem:

Theorem 3.1 The sequence of laws in $\mathcal{C}\left([0, T]^{d}\right)$ of the processes $\left\{X_{n}(t) ; t \in\right.$ $\left.[0, T]^{d}\right\}$ defined by (2.5) converges weakly to the law of $\left\{B_{t}^{\alpha} ; t \in[0, T]^{d}\right\}$.

In order to prove this result we have to check that the family of laws of the processes $\left\{X_{n}\right\}$ is tight and that any weakly convergent subsequence converges to the law of the $d$-parameter fractional Brownian sheet.

First of all we will see two technical lemmas. The first one will be useful to check the identification of the limit law.

Lemma 3.2 Consider $f_{1}, f_{2}, \ldots, f_{d} \in L^{2}([0, T])$. Then,

$$
E\left(\int_{[0, T]^{d}}\left(\prod_{i=1}^{d} f_{i}\left(u_{i}\right)\right) \theta_{n}(u) d u\right)^{2} \leq \prod_{i=1}^{d} \int_{0}^{T} f_{i}^{2}(x) d x .
$$

Proof: Notice that,

$$
\begin{aligned}
E\left(\int_{[0, T]^{d}}\right. & \left.\left(\prod_{i=1}^{d} f_{i}\left(u_{i}\right)\right) \theta_{n}(u) d u\right)^{2}= \\
& =E\left(\int_{[0, T]^{2 d}}\left(\prod_{i=1}^{d} f_{i}\left(u_{i}\right) f_{i}\left(v_{i}\right)\right) \theta_{n}(u) \theta_{n}(v) d u d v\right) \\
& =\int_{[0, T]^{2 d}}\left(\prod_{i=1}^{d} f_{i}\left(u_{i}\right) f_{i}\left(v_{i}\right)\right) E\left(\theta_{n}(u) \theta_{n}(v)\right) d u d v .
\end{aligned}
$$

But,

$$
\begin{aligned}
E\left(\theta_{n}(u) \theta_{n}(v)\right) & =n^{d} E\left[\left(\sum_{k \in \mathbb{N}^{d}} Z_{k} I_{[k-1, k)}(u \cdot n)\right)\left(\sum_{k \in \mathbb{N}^{d}} Z_{k} I_{[k-1, k)}(v \cdot n)\right)\right] \\
& =n^{d} \sum_{k \in \mathbb{N}^{d}} I_{[k-1, k)}(u \cdot n) I_{[k-1, k)}(v \cdot n),
\end{aligned}
$$

because for all $k \in \mathbb{N}^{d}$ we have that $E\left(Z_{k}\right)=0$ and $E\left(Z_{k}^{2}\right)=1$.

Using now that for all $a, b \in \mathbb{R}, a b \leq \frac{1}{2}\left(a^{2}+b^{2}\right)$,

$$
\begin{aligned}
E\left(\int_{[0, T]^{d}}\left(\prod_{i=1}^{d} f_{i}\left(u_{i}\right)\right) \theta_{n}(u) d u\right)^{2} & =\sum_{k \in \mathbb{N}^{d}} n^{d} \prod_{i=1}^{d}\left(\int_{0}^{T} f_{i}\left(u_{i}\right) I_{\left[k_{i}-1, k_{i}\right)}\left(u_{i} \cdot n\right) d u_{i}\right)^{2} \\
& \leq \prod_{i=1}^{d} \int_{0}^{T} f_{i}^{2}\left(u_{i}\right) d u_{i} .
\end{aligned}
$$

This finishes the proof of the lemma. 
We will prove the tightness of the laws using the following lemma:

Lemma 3.3 For any even number $m \in \mathbb{N}$, there exists a constant $C_{m}$ such that for any $s, t \in[0, T]^{d}$ with $s<t$

$$
\sup _{n} E\left[\Delta_{s} X_{n}(t)\right]^{m} \leq C_{m} \prod_{i=1}^{d}\left(t_{i}-s_{i}\right)^{m \alpha_{i}} .
$$

Proof: We have that

$$
\begin{aligned}
& E\left[\Delta_{s} X_{n}(t)\right]^{m}=E\left[\int_{[0, T]^{d}} \prod_{i=1}^{d}\left(K_{\alpha_{i}}\left(t_{i}, u_{i}\right)-K_{\alpha_{i}}\left(s_{i}, u_{i}\right)\right) \theta_{n}(u) d u\right]^{m} \\
& =\int_{[0, T]^{d m}} \prod_{j=1}^{m}\left[\prod_{i=1}^{d}\left(K_{\alpha_{i}}\left(t_{i}, u_{i}^{j}\right)-K_{\alpha_{i}}\left(s_{i}, u_{i}^{j}\right)\right)\right] E\left[\prod_{j=1}^{m} \theta_{n}\left(u^{j}\right)\right] d u^{1} \cdots d u^{m} .
\end{aligned}
$$

Observe that

$$
\begin{aligned}
& E\left[\prod_{j=1}^{m} \theta_{n}\left(u^{j}\right)\right]=n^{\frac{d m}{2}} E\left[\prod_{j=1}^{m}\left(\sum_{k \in \mathbb{N}^{d}} Z_{k} I_{[k-1, k)}\left(u^{j} \cdot n\right)\right)\right] \\
& =n^{\frac{d m}{2}} \sum_{k^{1}, \ldots, k^{m} \in \mathbb{N}^{d}} E\left(Z_{k^{1}} \cdots Z_{k^{m}}\right) I_{\left[k^{1}-1, k^{1}\right)}\left(u^{1} \cdot n\right) \cdots I_{\left[k^{m}-1, k^{m}\right)}\left(u^{m} \cdot n\right) .
\end{aligned}
$$

But notice that $E\left(Z_{k^{1}} \cdots Z_{k^{m}}\right)=0$ if for some $j \in\{1, \ldots, m\}, k^{j} \neq k^{l}$ for all $l \in\{1, \ldots, m\} \backslash\{j\}$, that is, if some variable $Z_{k^{j}}$ appears only once in the product $Z_{k^{1}} \cdots Z_{k^{m}}$.

On the other hand, since $E\left(\left|Z_{k}\right|^{m}\right)<\infty$ for all $k \in \mathbb{N}^{d}$ and $m \in \mathbb{N}$, $E\left(Z_{k^{1}} \cdots Z_{k^{m}}\right)$ is bounded for all $k^{1}, \ldots, k^{m} \in \mathbb{N}^{d}$. So,

$$
E\left[\prod_{j=1}^{m} \theta_{n}\left(u^{j}\right)\right] \leq n^{\frac{d m}{2}} C \sum_{\left(k^{1}, \ldots, k^{m}\right) \in A^{m}} I_{\left[k^{1}-1, k^{1}\right)}\left(u^{1} \cdot n\right) \cdots I_{\left[k^{m}-1, k^{m}\right)}\left(u^{m} \cdot n\right),
$$

with

$$
\begin{aligned}
& A^{m}=\left\{\left(k^{1}, \ldots, k^{m}\right) \in \mathbb{N}^{d m} ; \text { for all } l \in\{1, \ldots, m\}\right. \\
& \left.\qquad k^{l}=k^{j} \text { for some } j \in\{1, \ldots, m\} \backslash\{l\}\right\} .
\end{aligned}
$$

Notice that,

$$
\sum_{\left(k^{1}, \ldots, k^{m}\right) \in A^{m}} I_{\left[k^{1}-1, k^{1}\right)}\left(u^{1} \cdot n\right) \cdots I_{\left[k^{m}-1, k^{m}\right)}\left(u^{m} \cdot n\right) \leq I_{D^{m}}\left(u^{1}, \ldots, u^{m}\right),
$$

where

$$
\begin{aligned}
D^{m}:=\{ & \left(u^{1}, \ldots, u^{m}\right) \in[0, T]^{d m} ; \text { for all } l \in\{1, \ldots, m\} \text { there exists } \\
& \left.j \in\{1, \ldots, m\} \backslash\{l\} \text { such that }\left|u_{i}^{j}-u_{i}^{l}\right| \leq \frac{1}{n} \text { for all } i=1, \ldots, d\right\} .
\end{aligned}
$$


We can bound $I_{D^{m}}\left(u^{1}, \ldots, u^{m}\right)$ by a finite sum of products of indicators where in each product of indicators appear all the $d m$ variables $u_{1}^{1}, \ldots, u_{d}^{m}$ but each indicator concerns only two or three of them. Moreover, each variable appears only in one of the indicators of each product. So, expression (3.1) is equal to a sum of products of the following two kinds of terms:

(i) For some $l \neq j, l, j \in\{1, \ldots, m\}$,

$$
\begin{aligned}
C n^{d} \int_{[0, T]^{2 d}} & \prod_{i=1}^{d}\left(K_{\alpha_{i}}\left(t_{i}, u_{i}^{j}\right)-K_{\alpha_{i}}\left(s_{i}, u_{i}^{j}\right)\right) \prod_{i=1}^{d}\left(K_{\alpha_{i}}\left(t_{i}, u_{i}^{l}\right)-K_{\alpha_{i}}\left(s_{i}, u_{i}^{l}\right)\right) \\
& \times \prod_{i=1}^{d} I_{\left[0, \frac{1}{n}\right)}\left(\left|u_{i}^{j}-u_{i}^{l}\right|\right) d u^{j} d u^{l} .
\end{aligned}
$$

(ii) For some different $l, j, r \in\{1, \ldots, m\}$,

$$
\begin{aligned}
& C n^{\frac{3 d}{2}} \int_{[0, T]^{3 d}} \prod_{i=1}^{d}\left(K_{\alpha_{i}}\left(t_{i}, u_{i}^{j}\right)-K_{\alpha_{i}}\left(s_{i}, u_{i}^{j}\right)\right) \prod_{i=1}^{d}\left(K_{\alpha_{i}}\left(t_{i}, u_{i}^{l}\right)-K_{\alpha_{i}}\left(s_{i}, u_{i}^{l}\right)\right) \\
& \quad \times \prod_{i=1}^{d}\left(K_{\alpha_{i}}\left(t_{i}, u_{i}^{r}\right)-K_{\alpha_{i}}\left(s_{i}, u_{i}^{r}\right)\right) \prod_{i=1}^{d} I_{\left[0, \frac{1}{n}\right)}\left(\left|u_{i}^{j}-u_{i}^{l}\right|\right) \prod_{i=1}^{d} I_{\left[0, \frac{1}{n}\right)}\left(\left|u_{i}^{j}-u_{i}^{r}\right|\right) \\
& \quad \times \prod_{i=1}^{d} I_{\left[0, \frac{1}{n}\right)}\left(\left|u_{i}^{r}-u_{i}^{l}\right|\right) d u^{j} d u^{l} d u^{r} .
\end{aligned}
$$

Then, to prove the result it suffices to bound the first type of products by $C \prod_{i=1}^{d}\left(t_{i}-s_{i}\right)^{2 \alpha_{i}}$ and the second one by $C \prod_{i=1}^{d}\left(t_{i}-s_{i}\right)^{3 \alpha_{i}}$.

But using that for all $a, b \in \mathbb{R}, 2 a b \leq a^{2}+b^{2}$, we have that the first type of terms is bounded by,

$$
\begin{aligned}
C n^{d} \int_{[0, T]^{2 d}} \prod_{i=1}^{d} & \left(K_{\alpha_{i}}\left(t_{i}, u_{i}^{j}\right)-K_{\alpha_{i}}\left(s_{i}, u_{i}^{j}\right)\right)^{2} \prod_{i=1}^{d} I_{\left[0, \frac{1}{n}\right)}\left(\left|u_{i}^{j}-u_{i}^{l}\right|\right) d u^{j} d u^{l} \\
& \leq C \int_{[0, T]} \prod_{i=1}^{d}\left(K_{\alpha_{i}}\left(t_{i}, u_{i}^{j}\right)-K_{\alpha_{i}}\left(s_{i}, u_{i}^{j}\right)\right)^{2} d u^{j} \\
& \leq C \prod_{i=1}^{d}\left(E\left(B_{t_{i}}^{\alpha_{i}}-B_{s_{i}}^{\alpha_{i}}\right)^{2}\right) \\
& =C \prod_{i=1}^{d}\left(t_{i}-s_{i}\right)^{2 \alpha_{i}}
\end{aligned}
$$

where for all $i \in\{1,2, \ldots, d\},\left\{B^{\alpha_{i}}\right\}$ is a standard fractional Brownian motion of parameter $\alpha_{i}$. 
On the other hand, we can study the terms of type $(i i)$ in the following way:

$$
\begin{aligned}
& \int_{[0, T]^{3 d}} \prod_{i=1}^{d}\left(K_{\alpha_{i}}\left(t_{i}, u_{i}^{j}\right)-K_{\alpha_{i}}\left(s_{i}, u_{i}^{j}\right)\right) \prod_{i=1}^{d}\left(K_{\alpha_{i}}\left(t_{i}, u_{i}^{l}\right)-K_{\alpha_{i}}\left(s_{i}, u_{i}^{l}\right)\right) \\
& \quad \times \prod_{i=1}^{d}\left(K_{\alpha_{i}}\left(t_{i}, u_{i}^{r}\right)-K_{\alpha_{i}}\left(s_{i}, u_{i}^{r}\right)\right) \prod_{i=1}^{d} I_{\left[0, \frac{1}{n}\right)}\left(\left|u_{i}^{j}-u_{i}^{l}\right|\right) \prod_{i=1}^{d} I_{\left[0, \frac{1}{n}\right)}\left(\left|u_{i}^{j}-u_{i}^{r}\right|\right) \\
& \quad \times \prod_{i=1}^{d} I_{\left[0, \frac{1}{n}\right)}\left(\left|u_{i}^{r}-u_{i}^{l}\right|\right) d u^{j} d u^{l} d u^{r} \\
& =\int_{[0, T]^{2 d}} \prod_{i=1}^{d}\left(K_{\alpha_{i}}\left(t_{i}, u_{i}^{j}\right)-K_{\alpha_{i}}\left(s_{i}, u_{i}^{j}\right)\right) \prod_{i=1}^{d} I_{\left[0, \frac{1}{n}\right)}\left(\left|u_{i}^{j}-u_{i}^{l}\right|\right) \\
& \quad \times \int_{[0, T]^{d}} \prod_{i=1}^{d}\left(K_{\alpha_{i}}\left(t_{i}, u_{i}^{l}\right)-K_{\alpha_{i}}\left(s_{i}, u_{i}^{l}\right)\right) \prod_{i=1}^{d}\left(K_{\alpha_{i}}\left(t_{i}, u_{i}^{r}\right)-K_{\alpha_{i}}\left(s_{i}, u_{i}^{r}\right)\right) \\
& \quad \times \prod_{i=1}^{d} I_{\left[0, \frac{1}{n}\right)}\left(\left|u_{i}^{j}-u_{i}^{r}\right|\right) \prod_{i=1}^{d} I_{\left[0, \frac{1}{n}\right)}\left(\left|u_{i}^{r}-u_{i}^{l}\right|\right) d u^{r} d u^{l} d u^{j},
\end{aligned}
$$

and the last expression can be bounded by

$$
\begin{aligned}
& \left(\int_{[0, T]^{2 d}} \prod_{i=1}^{d}\left(K_{\alpha_{i}}\left(t_{i}, u_{i}^{j}\right)-K_{\alpha_{i}}\left(s_{i}, u_{i}^{j}\right)\right)^{2} \prod_{i=1}^{d} I_{\left[0, \frac{1}{n}\right)}\left(\left|u_{i}^{j}-u_{i}^{l}\right|\right) d u^{l} d u^{j}\right)^{\frac{1}{2}} \\
& \times\left(\int _ { [ 0 , T ] ^ { 2 d } } \left(\int_{[0, T]^{d}} \prod_{i=1}^{d}\left(K_{\alpha_{i}}\left(t_{i}, u_{i}^{l}\right)-K_{\alpha_{i}}\left(s_{i}, u_{i}^{l}\right)\right) \prod_{i=1}^{d}\left(K_{\alpha_{i}}\left(t_{i}, u_{i}^{r}\right)-K_{\alpha_{i}}\left(s_{i}, u_{i}^{r}\right)\right)\right.\right. \\
& \left.\left.\times \prod_{i=1}^{d} I_{\left[0, \frac{1}{n}\right)}\left(\left|u_{i}^{j}-u_{i}^{r}\right|\right) \prod_{i=1}^{d} I_{\left[0, \frac{1}{n}\right)}\left(\left|u_{i}^{r}-u_{i}^{l}\right|\right) d u^{r}\right)^{2} d u^{l} d u^{j}\right)^{\frac{1}{2}} .
\end{aligned}
$$

As for the terms of type $(i)$, we have that

$$
\begin{array}{r}
\left(\int_{[0, T]^{2 d}} \prod_{i=1}^{d}\left(K_{\alpha_{i}}\left(t_{i}, u_{i}^{j}\right)-K_{\alpha_{i}}\left(s_{i}, u_{i}^{j}\right)\right)^{2} \prod_{i=1}^{d} I_{\left[0, \frac{1}{n}\right)}\left(\left|u_{i}^{j}-u_{i}^{l}\right|\right) d u^{l} d u^{j}\right)^{\frac{1}{2}} \\
\leq \frac{1}{n^{\frac{d}{2}}} \prod_{i=1}^{d}\left(t_{i}-s_{i}\right)^{\alpha_{i}}
\end{array}
$$


On the other hand,

$$
\begin{aligned}
& \left(\int _ { [ 0 , T ] ^ { 2 d } } \left(\int_{[0, T]^{d}} \prod_{i=1}^{d}\left(K_{\alpha_{i}}\left(t_{i}, u_{i}^{l}\right)-K_{\alpha_{i}}\left(s_{i}, u_{i}^{l}\right)\right) \prod_{i=1}^{d}\left(K_{\alpha_{i}}\left(t_{i}, u_{i}^{r}\right)-K_{\alpha_{i}}\left(s_{i}, u_{i}^{r}\right)\right)\right.\right. \\
& \left.\left.\quad \times \prod_{i=1}^{d} I_{\left[0, \frac{1}{n}\right)}\left(\left|u_{i}^{j}-u_{i}^{r}\right|\right) \prod_{i=1}^{d} I_{\left[0, \frac{1}{n}\right)}\left(\left|u_{i}^{r}-u_{i}^{l}\right|\right) d u^{r}\right)^{2} d u^{l} d u^{j}\right)^{\frac{1}{2}} \\
& =\left(\int_{[0, T]^{4 d}} \prod_{i=1}^{d}\left(K_{\alpha_{i}}\left(t_{i}, u_{i}^{l}\right)-K_{\alpha_{i}}\left(s_{i}, u_{i}^{l}\right)\right)^{2} \prod_{i=1}^{d}\left(K_{\alpha_{i}}\left(t_{i}, u_{i}^{r_{1}}\right)-K_{\alpha_{i}}\left(s_{i}, u_{i}^{r_{1}}\right)\right)\right. \\
& \quad \times \prod_{i=1}^{d}\left(K_{\alpha_{i}}\left(t_{i}, u_{i}^{r_{2}}\right)-K_{\alpha_{i}}\left(s_{i}, u_{i}^{r_{2}}\right)\right) \prod_{i=1}^{d} I_{\left[0, \frac{1}{n}\right)}\left(\left|u_{i}^{j}-u_{i}^{r_{1}}\right|\right) \prod_{i=1}^{d} I_{\left[0, \frac{1}{n}\right)}\left(\left|u_{i}^{r_{1}}-u_{i}^{l}\right|\right) \\
& \left.\quad \times \prod_{i=1}^{d} I_{\left[0, \frac{1}{n}\right)}\left(\left|u_{i}^{j}-u_{i}^{r_{2}}\right|\right) \prod_{i=1}^{d} I_{\left[0, \frac{1}{n}\right)}\left(\left|u_{i}^{r_{2}}-u_{i}^{l}\right|\right) d u^{l} d u^{j} d u^{r_{1}} d u^{r_{2}}\right)^{\frac{1}{2}} \\
& \leq\left(\frac{1}{n^{d}} \int_{[0, T]^{3 d}} \prod_{i=1}^{d}\left(K_{\alpha_{i}}\left(t_{i}, u_{i}^{l}\right)-K_{\alpha_{i}}\left(s_{i}, u_{i}^{l}\right)\right)^{2} \prod_{i=1}^{d}\left(K_{\alpha_{i}}\left(t_{i}, u_{i}^{r_{1}}\right)-K_{\alpha_{i}}\left(s_{i}, u_{i}^{r_{1}}\right)\right)\right. \\
& \quad \times \prod_{i=1}^{d}\left(K_{\alpha_{i}}\left(t_{i}, u_{i}^{r_{2}}\right)-K_{\alpha_{i}}\left(s_{i}, u_{i}^{r_{2}}\right)\right) \prod_{i=1}^{d} I_{\left[0, \frac{1}{n}\right)}\left(\left|u_{i}^{r_{1}}-u_{i}^{l}\right|\right) \\
& \left.\quad \times \prod_{i=1}^{d} I_{\left[0, \frac{1}{n}\right)}\left(\left|u_{i}^{r_{2}}-u_{i}^{l}\right|\right) d u^{l} d u^{r_{1}} d u^{r_{2}}\right)^{\frac{1}{2}},
\end{aligned}
$$

and this expression can be majorized as follows

$$
\begin{aligned}
& \left(\frac{1}{n^{d}} \int_{[0, T]^{3 d}} \prod_{i=1}^{d}\left(K_{\alpha_{i}}\left(t_{i}, u_{i}^{l}\right)-K_{\alpha_{i}}\left(s_{i}, u_{i}^{l}\right)\right)^{2} \prod_{i=1}^{d}\left(K_{\alpha_{i}}\left(t_{i}, u_{i}^{r_{1}}\right)-K_{\alpha_{i}}\left(s_{i}, u_{i}^{r_{1}}\right)\right)^{2}\right. \\
& \left.\times \prod_{i=1}^{d} I_{\left[0, \frac{1}{n}\right)}\left(\left|u_{i}^{r_{2}}-u_{i}^{l}\right|\right) d u^{l} d u^{r_{1}} d u^{r_{2}}\right)^{\frac{1}{2}} \\
& \leq\left(\frac{1}{n^{2 d}} \int_{[0, T]^{2 d}} \prod_{i=1}^{d}\left(K_{\alpha_{i}}\left(t_{i}, u_{i}^{l}\right)-K_{\alpha_{i}}\left(s_{i}, u_{i}^{l}\right)\right)^{2} \times\right. \\
& \left.\quad \times \prod_{i=1}^{d}\left(K_{\alpha_{i}}\left(t_{i}, u_{i}^{r_{1}}\right)-K_{\alpha_{i}}\left(s_{i}, u_{i}^{r_{1}}\right)\right)^{2} d u^{l} d u^{r_{1}}\right)^{\frac{1}{2}} \\
& =\left(\frac{1}{n^{2 d}} \prod_{i=1}^{d}\left(t_{i}-s_{i}\right)^{4 \alpha_{i}}\right)^{\frac{1}{2}}=\frac{1}{n^{d}} \prod_{i=1}^{d}\left(t_{i}-s_{i}\right)^{2 \alpha_{i}}
\end{aligned}
$$

using again the bound for the terms of type (i). 
So, we have obtained that the terms of type $(i i)$ can be bounded by

$$
C \prod_{i=1}^{d}\left(t_{i}-s_{i}\right)^{3 \alpha_{i}}
$$

and this finishes the proof of the lemma.

\subsection{Proof of Theorem 3.1}

We need to prove that the family of laws of the processes $\left\{X_{n}\right\}$ is tight and we have to identify the limit law of any convergent subsequence as the law of a $d$-parameter fractional Brownian sheet.

Using the criterion given by Bickel and Wichura (1971) and that the processes $X_{n}$ are null when some coordinate equals zero the tightness is a consequence of Lemma 3.3.

To check the identification of the limit law we have to prove the convergence of the finite dimensional distributions of $\left\{X_{n}\right\}$ to those of the $d$ parameter fractional Brownian sheet, $\left\{B^{\alpha}\right\}$.

It suffices to prove that for any $k \in \mathbb{N}, a_{1}, \ldots, a_{k} \in \mathbb{R}$ and $s^{1}, \ldots, s^{k} \in$ $[0, T]^{d}$ the characteristic function of

$$
\sum_{j=1}^{k} a_{j} X_{n}\left(s^{j}\right)
$$

converges to the characteristic function of

$$
\sum_{j=1}^{k} a_{j} B_{s^{j}}^{\alpha},
$$

when $n$ tends to infinity.

Consider for any $s^{j} \in[0, T]^{d}$ sequences $\left\{\rho_{1}^{j, l}\right\}, \ldots,\left\{\rho_{d}^{j, l}\right\}$ of elementary functions such that for any $i \in\{1, \ldots, d\},\left\{\rho_{i}^{j, l}\right\}$ converges in $L^{2}([0,1])$ to $K_{\alpha_{i}}\left(s_{i}^{j}, \cdot\right)$ as $l$ tends to infinity.

We introduce also the following notations

$$
\begin{aligned}
X_{n}^{j, l} & =\int_{[0, T]^{d}} \prod_{i=1}^{d} \rho_{i}^{j, l}\left(u_{i}\right) \theta_{n}(u) d u, \\
X^{j, l} & =\int_{[0, T]^{d}} \prod_{i=1}^{d} \rho_{i}^{j, l}\left(u_{i}\right) d W_{u},
\end{aligned}
$$

where $\left\{W_{u} ; u \in[0, T]^{d}\right\}$ is a standard $d$-parameter Wiener process. 
Then for any $\lambda \in \mathbb{R}$,

$$
\begin{aligned}
\left|E\left[\exp \left(i \lambda \sum_{j} a_{j} X_{n}\left(s^{j}\right)\right)\right]-\bar{E}\left[\exp \left(i \lambda \sum_{j} a_{j} B_{s_{j}}^{\alpha}\right)\right]\right| \\
\leq\left|E\left[\exp \left(i \lambda \sum_{j} a_{j} X_{n}\left(s^{j}\right)\right)-\exp \left(i \lambda \sum_{j} a_{j} X_{n}^{j, l}\right)\right]\right| \\
+\left|E\left[\exp \left(i \lambda \sum_{j} a_{j} X_{n}^{j, l}\right)\right]-\bar{E}\left[\exp \left(i \lambda \sum_{j} a_{j} X^{j, l}\right)\right]\right| \\
+\left|\bar{E}\left[\exp \left(i \lambda \sum_{j} a_{j} X^{j, l}\right)-\exp \left(i \lambda \sum_{i} a_{j} B_{s_{j}}^{\alpha}\right)\right]\right| .
\end{aligned}
$$

By the mean value theorem the first summand can be bounded by

$$
C \max _{j}\left\{E\left|X_{n}\left(s^{j}\right)-X_{n}^{j, l}\right|\right\} .
$$

And we can bound this expectation in the following way:

$$
\begin{aligned}
& E\left|X_{n}\left(s^{j}\right)-X_{n}^{j, l}\right| \\
& =E\left|\int_{[0, T]^{d}}\left(\prod_{i=1}^{d} K_{\alpha_{i}}\left(s_{i}^{j}, u_{i}\right)-\prod_{i=1}^{d} \rho_{i}^{j, l}\left(u_{i}\right)\right) \theta_{n}(u) d u\right| \\
& =E\left|\int_{[0, T]^{d}}\left(K_{\alpha_{1}}\left(s_{1}^{j}, u_{1}\right)-\rho_{1}^{j, l}\left(u_{1}\right)\right) K_{\alpha_{2}}\left(s_{2}^{j}, u_{2}\right) \cdots K_{\alpha_{d}}\left(s_{d}^{j}, u_{d}\right) \theta_{n}(u) d u\right| \\
& +E\left|\int_{[0, T]^{d}} \rho_{1}^{j, l}\left(u_{1}\right)\left(K_{\alpha_{2}}\left(s_{2}^{j}, u_{2}\right)-\rho_{2}^{j, l}\left(u_{2}\right)\right) K_{\alpha_{3}}\left(s_{3}^{j}, u_{3}\right) \cdots K_{\alpha_{d}}\left(s_{d}^{j}, u_{d}\right) \theta_{n}(u) d u\right| \\
& +\cdots+E\left|\int_{[0, T]^{d}} \rho_{1}^{j, l}\left(u_{1}\right) \cdots \rho_{d-1}^{j, l}\left(u_{d-1}\right)\left(K_{\alpha_{d}}\left(s_{d}^{j}, u_{d}\right)-\rho_{d}^{j, l}\left(u_{d}\right)\right) \theta_{n}(u) d u\right| \\
& \leq\left(\int_{[0, T]^{d}}\left(K_{\alpha_{1}}\left(s_{1}^{j}, u_{1}\right)-\rho_{1}^{j, l}\left(u_{1}\right)\right)^{2}\left(K_{\alpha_{2}}\left(s_{2}^{j}, u_{2}\right)\right)^{2} \cdots\left(K_{\alpha_{d}}\left(s_{d}^{j}, u_{d}\right)\right)^{2} d u\right)^{\frac{1}{2}} \\
& +\left(\int_{[0, T]^{d}}\left(\rho_{1}^{j, l}\left(u_{1}\right)\right)^{2}\left(K_{\alpha_{2}}\left(s_{2}^{j}, u_{2}\right)-\rho_{2}^{j, l}\left(u_{2}\right)\right)^{2}\left(K_{\alpha_{3}}\left(s_{3}^{j}, u_{3}\right)\right)^{2} \times \cdots\right. \\
& \left.\quad \ldots \times\left(K_{\alpha_{d}}\left(s_{d}^{j}, u_{d}\right)\right)^{2} d u\right)^{\frac{1}{2}} \\
& +\cdots+\left(\int_{[0, T]^{d}}\left(\rho_{1}^{j, l}\left(u_{1}\right)\right)^{2} \ldots\left(\rho_{d-1}^{j, l}\left(u_{d-1}\right)\right)^{2}\left(K_{\alpha_{d}}\left(s_{d}^{j}, u_{d}\right)-\rho_{d}^{j, l}\left(u_{d}\right)\right)^{2} d u\right)^{\frac{1}{2}}
\end{aligned}
$$

where we have applied Lemma 3.2. 
Observe that each sumand is a product of bounded integrals where one of them is equal to

$$
\int_{[0, T]}\left(K_{\alpha_{i}}\left(s_{i}^{j}, u_{i}\right)-\rho_{i}^{j, l}\left(u_{i}\right)\right)^{2} d u_{i}
$$

for some $i \in\{1, \ldots, d\}$. This integral, if $l$ is large enough, can be made arbitrarily small independently of $n$.

Since $\left\{\rho_{i}^{j, l}\right\}$ are elementary functions, the random variables $X_{n}^{j, l}$ are linear combinations of increments of the process

$$
Y_{n}(s)=\int_{\left[0, s_{1}\right] \times \cdots \times\left[0, s_{d}\right]} \theta_{n}(u) d u .
$$

Moreover, the laws of these processes converge weakly, in $\mathcal{C}\left([0, T]^{d}\right)$, to the law of the $d$-parameter Wiener process from the functional invariance principle (see, for instance, Wichura, 1969). Then, the linear combinations of the increments of $Y_{n}$ will converge in law to $X^{j, l}$, and so, fixed $l \in \mathbb{N}$, the second summand of (3.2) tends to zero when $n \rightarrow \infty$.

Finally, the third summand of (3.2) is bounded by

$$
\begin{aligned}
C \max _{j} & \left\{\bar{E}\left|X^{j, l}-B_{s_{j}}^{\alpha}\right|\right\} \\
& =C \max _{j}\left\{\bar{E}\left|\int_{[0, T]^{d}}\left(\prod_{i=1}^{d} \rho_{i}^{j, l}\left(u_{i}\right)-\prod_{i=1}^{d} K_{\alpha_{i}}\left(s_{i}, u_{i}\right)\right) d W_{u}\right|\right\} \\
& \leq C \max _{j}\left(\int_{[0, T]^{d}}\left(\prod_{i=1}^{d} \rho_{i}^{j, l}\left(u_{i}\right)-\prod_{i=1}^{d} K_{\alpha_{i}}\left(s_{i}, u_{i}\right)\right)^{2} d u\right)^{\frac{1}{2}},
\end{aligned}
$$

using the properties of the stochastic integral. And this last expression converges to zero when $l \uparrow \infty$ independently of $n$ by the same arguments used for the first summand of (3.2).

The proof of Theorem (3.1) is now complete.

\section{References}

[1] Alòs, E., Mazet, O., Nualart, D.: Stochastic calculus with respect to Gaussian processes. Ann. Probab. 29 (2001), no. 2, 766-801.

[2] Bardina, X., Jolis, M., Tudor, C. A.: Convergence in law to the multiple fractional integral. Stochastic Process. Appl. 105 (2003), no. 2, 315-344.

[3] Bardina, X., Jolis, M., Tudor, C. A.: Weak convergence to the fractional Brownian sheet and other two-parameter Gaussian processes. Statist. Probab. Lett. 65 (2003), no. 4, 317-329. 
[4] Bickel, P. J., Wichura, M. J.: Convergence criteria for multiparameter stochastic processes and some applications. Ann. Math. Statist. 42 (1971), 1656-1670.

[5] Čencov, N. N.: Wiener random fields depending on several parameters. Dokl. Akad. Nauk SSSR (N.S.) 106 (1956), 607-609.

[6] Ciesielski, Z., Kamont, A.: Levy's fractional Brownian random field and function spaces. Acta Sci. Math. (Szeged) 60 (1995), no. 1-2, 99-118.

[7] Davydov, J. A.: The invariance principle for stationary processes. Teor. Verojatnost. i Primenen. 15 (1970), 498-509.

[8] Delgado, R., Jolis, M.: Weak approximation for a class of Gaussian processes. J. Appl. Probab. 37 (2000), no. 2, 400-407.

[9] Hult, H.: Approximating some Volterra type stochastic integrlas with applications to parameter estimation. Stochastic Process. Appl. 105 (2003), no. $1,1-32$.

[10] Kamont, A.: On the fractional anisotropic Wiener field. Probab. Math. Statist. 16 (1996), no. 1, 85-98.

[11] Mandelbrot, B. B., Van Ness, J. W.: Fractional Brownian motion, fractional noises and applications. SIAM Rev. 10 (1968), no. 4, 422-437.

[12] Pipiras, V., Taqqu, M. S.: Convergence of the Weierstrass-Mandelbrot process to fractional Brownian motion. Fractals 8 (2000), no. 4, 369-384.

[13] Sottinen, T.: Fractional Brownian motion, random walks and binary market models. Finance Stoch. 5 (2001), no. 3, 343-355.

[14] Wichura, M. J.: Inequalities with applications to the weak convergence of random processes with multi-dimensional time parameters. Ann. Math. Statist. 40 (1969), 681-687.

Recibido: 8 de octubre de 2003

Xavier Bardina

Departament de Matemàtiques Universitat Autònoma de Barcelona 08193-Bellaterra (Barcelona), Spain

bardina@mat.uab.es

Carme Florit

Departament de Matemàtiques Universitat Autònoma de Barcelona 08193-Bellaterra (Barcelona), Spain

cflorit@mat.uab.es

$\overline{\text { First author is partially supported }}$ by DGES Grants BFM2003-01345, BFM2003-00261 and by CIRIT Grant 2001SGR00174. 